\title{
Viewpoint
}

\section{Guiding future high-field magnet design by better predicting and mitigating screening current-induced stress and strain}

\author{
Mark D Ainslie ${ }^{1}$ \\ ${ }^{1}$ Bulk Superconductivity Group, Department of Engineering, University of Cambridge, United \\ Kingdom \\ mark.ainslie@eng.cam.ac.uk
}

This is a viewpoint on the Letter by Yufan Yan et al (2020 Supercond. Sci. Technol. 33 05LT02).

High-field magnets are essential across a range of scientific disciplines. They are used in particle accelerators for high-energy physics to understand the most fundamental constituents of matter and how these interact, to ultimately understand the universe around us. Higher magnetic fields lead to improvements in the sensitivity and resolution of nuclear magnetic resonance (NMR) and magnetic resonance imaging (MRI) - powerful tools used to study materials noninvasively at various levels. High-field magnets are also an essential component of magnetic confinement fusion devices, long touted as the clean and sustainable energy source needed to mitigate climate change and meet our ever-increasing energy demands.

Significant advances in high-field magnet technology continue to be made as scientists seek improved performance and ever-higher magnetic fields. Magnet designers face several difficult technological challenges when developing such magnets in order to satisfy several (often competing) constraints, including electromagnetic, thermal, structural and economic ones. This is notwithstanding the additional constraints specific to a particular application.

Since high-temperature superconducting (HTS) materials were discovered in the 1980s, there has been continued interest in exploiting them in high-field magnets. HTS materials have significant advantages over other materials: they are able to carry higher currents in the presence of a magnetic field at higher operating temperatures than other practical superconducting materials, offer increased thermal stability with a larger temperature margin and have improved mechanical properties. Thus, HTS magnets offer the possibility of higher magnetic fields with a smaller magnet footprint, simpler cryogenics with the possibility of cryogen-free operation, increased stability (from several viewpoints, including thermal, mechanical and noise) and lower power consumption (especially in comparison to resistive and hybrid magnets). Continued advances in the manufacturing of HTS conductors have resulted in the commercial availability - from several manufacturers worldwide - of the long lengths (100s of $\mathrm{m}$ to $\mathrm{km}+$ ) of high-quality conductor required to wind a large-scale HTS magnet.

The maximum field achieved in conventional low-temperature superconducting (LTS) magnets has not exceeded $23.5 \mathrm{~T}$, limited by the upper critical fields of the $\mathrm{NbTi}$ and $\mathrm{Nb}_{3} \mathrm{Sn}$ conductors used. In December 2017, a world-record 32 T was achieved by the National High 
Magnetic Field Laboratory (NHFML) in the USA in an all-superconducting magnet consisting of an inner HTS magnet and an outer LTS magnet [1]. Very recently, in February 2020, researchers at the Chinese Academy of Sciences (CAS) in China reported a new world-record 32.35 T with a similar configuration but using no-insulation (NI) HTS insert coils [2]. It should be noted that higher fields can be produced by resistive (e.g., NHFML's 41.5 T resistive magnet [3]) or hybrid (e.g., NHFML's 45 T hybrid magnet [4]) magnets, but these require 10s of MW of power to operate [5]. However, it is fully expected that these records will continue to be broken by more advanced all-superconducting magnets in the future - a new record DC field of $45.5 \mathrm{~T}$, achieved in a hybrid magnet using an NI HTS insert coil, was reported in June 2019 [6] for example - although these large-scale magnet projects do require significant investments in time, effort and funding $[7,8]$.

There are several major technical challenges for high-field HTS magnet designers $[5,8]$, including 1) the screening current-induced magnetic field, and its associated stress/strain; 2) fatigue degradation of the conductor, and associated degradation of coil performance, during cyclic operation; 3) quench protection; and 4) in the case of $\mathrm{NI}$ coils, magnetic field delay [9].

The work of Yan et al [10] addresses this first challenge - the screening current-induced magnetic field - and they carry out a comprehensive study on the strain distribution in an HTS magnet using experimental, numerical simulation and analytical techniques.

When an HTS magnet is energised and/or in a background magnetic field, some of the magnet field penetrates into the conductor, resulting in persistent screening currents being induced in the winding. These screening currents result in spatial distortion and temporal drift of the field. Thus, they can be a major problem for particle accelerators and NMR/MRI applications, where there are strict requirements for spatial homogeneity and temporal stability. Screening currents also create additional high stresses in the magnet that can result in irreversible degradation of the conductor/magnet performance and sometimes catastrophic damage $[5,6,11]$. Hence, understanding, predicting and mitigating the screening current in HTS magnets and its associated stress/strain is of vital importance.

In [10], Yan et al have designed a bespoke experimental setup consisting of an LTS background magnet, capable of providing up to $9 \mathrm{~T}$, and a small HTS insert coil, wound from $10 \mathrm{~mm}$ wide $\mathrm{REBa}_{2} \mathrm{Cu}_{3} \mathrm{O}_{7-\mathrm{x}}$ (REBCO) $\mathrm{HTS}$ coated conductor. Low-temperature strain gauges are used to measure the strain in the HTS magnet while it is energised. A numerical model based on the axisymmetric $\boldsymbol{T}$ - $\boldsymbol{A}$ formulation $[12,13]$, which efficiently tackles the problem of simulating superconductors with a very high aspect ratio by treating the superconductor as a $1 \mathrm{D}$ object, is validated using these experimental results. The authors also propose a simple analytical equation to estimate the component of the Lorentz force in the radial direction that could be most problematic for the coil, based on the axial field (background field + insert coil field) and the critical current density, which is dependent on this field as well as the radial field.

The model is then used to explore two methods to mitigate screening currents: filamentation of the HTS conductor and current sweep reversal (CSR). The deleterious effect 
of screening currents was actually first encountered in the 1960s during the development of LTS magnets, but the introduction of twisted, multifilamentary LTS conductors resulted in a dramatic reduction in the negative effects of screening currents [8]. This is more difficult to achieve with HTS conductors due to their different architecture, but striation of the conductor into narrow filaments is possible, which can also reduce hysteretic AC loss [14]. Yan et al use their model to show that screening current-induced strain can be dramatically reduced by using a striated multifilamentary HTS conductor. They also show that the CSR method could reduce the strain at the operating current but point out that the maximum strain still remains high for overshoot currents.

This work provides a step forward in high-field magnet design that should be an important stepping stone towards the generation of higher and higher continuous magnetic fields in the future.

\section{References}

[1] National High Magnetic Field Laboratory, 32 Tesla All-Superconducting Magnet [online] https://nationalmaglab.org/magnet-development/magnet-science-technology/magnetprojects/32-tesla-scm

[2] Liu J et al 2020 World record 32.35 tesla direct-current magnetic field generated with an all-superconducting magnet Supercond. Sci. Technol. 33 03LT01

[3] Toth J and Bole S T 2018 Design, construction, and first testing of a $41.5 \mathrm{~T}$ all-resistive magnet at the NHMFL in Tallahassee IEEE Trans. Appl. Supercond. 284300104

[4] Miller J R 2003 The NHMFL 45-T hybrid magnet system: Past, present, and future IEEE Trans. Appl. Supercond. 13 1385-90

[5] Bai $\mathrm{H}$ et al 2020 The $40 \mathrm{~T}$ superconducting magnet project at the National High Magnetic Field Laboratory IEEE Trans. Appl. Supercond. 304300405

[6] Hahn S et al 2019 45.5-tesla direct-current magnetic field generated with a hightemperature superconducting magnet Nature 570 496-9

[7] Pugnat P and Schneider-Muntau H J 2014 Hybrid magnets-Past, present, and future IEEE Trans. Appl. Supercond. 244300106

[8] Maeda $\mathrm{H}$ and Yanagisawa Y 2014 Recent developments in high-temperature superconducting magnet technology IEEE Trans. Appl. Supercond. 244602412

[9] Suetomi Y et al 2019 A novel winding method for a no-insulation layer-wound REBCO coil to provide a short magnetic field delay and self-protect characteristics Supercond. Sci.

Technol. 32045003

[10] Yan Y et al 2020 Screening current effect on the stress and strain distribution in REBCO high-field magnets: experimental verification and numerical analysis Supercond. Sci.

Technol. 33 05LT02

[11] Ma D X et al 2013 Degradation of REBCO conductors caused by the screening current Supercond. Sci. Technol. 26105018

[12] Zhang H, Zhang M and Yuan W 2016 An efficient 3D finite element method model based on the T-A formulation for superconducting coated conductors Supercond. Sci.

Technol. $\mathbf{3 0} 024005$ 
[13] Liang F et al 2017 A finite element model for simulating second generation high temperature superconducting coils/stacks with large number of turns J. Appl. Phys. 122 043903

[14] Ainslie M D 2019 Reducing AC losses in high-temperature superconducting coatedconductor wires towards more efficient superconducting electric power applications Supercond. Sci. Technol. 32030501 